\title{
Disseminated Tuberculosis Causing Addison's Disease in an Adult Diabetic Patient
}

\author{
NK QURESHI ${ }^{\mathrm{a}}$, ZA LATIF ${ }^{\mathrm{b}}$
}

Summary:

Tuberculosis may affect many of the endocrine glands including the hypothalamus, pituitary, thyroid with adrenal gland being the commonest. We describe a patient presented with recurrent spontaneous hypoglycemia, hypotension, fever, weight loss and cough. Along with this clinical picture, high ACTH in the face of low cortisol was compatible with diagnosis of Addison's disease. Rapid ACTH stimulation test affirmed the diagnosis. Disseminated TB affecting adrenal glands was supported by CXR, USG CT Abdomen and FNAC. Adrenal Crisis was led by add-on bacterial

\section{Introduction:}

Primary Adrenal insufficiency, which bears his name, was first described by Thomas Addison in $1855 .{ }^{1}$ In those days, tuberculosis was the most common cause of bilateral adrenal destruction leading to its insufficiency. ${ }^{1}$ Now-a-days, tuberculosis(TB) accounts for only 7 to 20 percent of cases and autoimmune adrenocortical atrophy and tuberculosis together accounts for nearly 90 percent cases, with the remainder being caused by other diseases e.g.; histoplasmosis, blastomycosis, cryptococcosis, sarcoidosis, metastases, lymphoma, adrenal hemorrhage, infarction, or drugs. ${ }^{2,3}$ Though overall incidence of Addison's disease (AD) has decreased, disseminated tuberculosis and fungal infections are still major causes of adrenal insufficiency in populations with a high prevalence of these diseases. ${ }^{4}$ At present, in developed countries most cases of $\mathrm{AD}$ are caused by autoimmune destruction and in developing countries TB is the leading aetiology. ${ }^{5,6}$ Adrenal insufficiency may manifest clinically in different ways

a. Dr. Nazmul Kabir Qureshi, Specialist, Department of Medicine, Endocrinology \& Gastroenterology, United Hospital, Dhaka, Bangladesh

b. Prof. Zafar Ahmed Latif, Professor and Head, Department of Endocrinology, Ibrahim Medical College \& BIRDEM hospital, Dhaka, Bangladesh

Address of Correspondence: Dr. Nazmul Kabir Qureshi MD(EM), Specialist, Department of Medicine, Endocrinology \& Gastroenterology, United Hospital Limited, Dhaka, Bangladesh, Phone: 01711671504, 01674082174, Email: qureshinaz@yahoo.com Received: 8 December, 2013 Accepted: 10 October, 2014 pneumonia. Patient made good recovery with treatment for adrenal crisis and subsequently with standard antitubercular regimen and steroid replacement therapy. Tuberculosis, although uncommon but potentially devastating cause of adrenal failure, merits consideration when fever, weight loss, gastrointestinal symptoms, hyponatremia, hyperkalemia are observed in patients with features of active tuberculosis or past history of tuberculosis.

Key words: Addison's disease (AD), Tuberculosis (TB).

(J Banagladesh Coll Phys Surg 2015; 33: 44-47)

as generalized weakness, fatigue, poor apatite, weight loss, diarrhea, steatorrhoea, fever, dizziness etc. ${ }^{7}$ "Adrenal crisis" which occur during stress like infection, surgery or trauma, may manifest as hypotension, hyponatremia, hyperkalemia and/or hypoglycemia even shock. Nomura et al. ${ }^{4}$ have reported that $92.9 \%$ of patients with adrenal TB may have a previous history of lung and/or pleural TB with mean $32( \pm 15)$ years of interval. The symptoms of primary Addison's disease manifest when more than $90 \%$ of the adrenal glands have been destroyed. ${ }^{8}$ For this reason Addison's disease due to TB manifests relatively late in life, predominantly in persons aged 40 to 60 years. ${ }^{4}$ The aim of this report is to describe a case of Addison's disease caused by disseminated tuberculosis which presented with a crisis lead by add-on bacterial pneumonia.

\section{Case Report:}

In January 2010, a 45-year-old Bangladeshi diabetic male previously on insulin presented at BIRDEM for evaluation of recurrent documented spontaneous hypoglycemia and hypotension leading to syncope on several occasions during past 4 months. History of missed, delayed meal, recent change of insulin dose, vomiting, diarrhea or any other inciting causes for hypoglycemia or hypotension were negative. On enquiry, he mentioned of $6 \mathrm{~kg}$ weight loss with persistent low grade fever with evening rise of temperature and paroxysmal productive cough which showed poor response to several courses of oral and intravenous antibiotics. Previous tubercular infection and history of 
close contact with patient with tuberculosis were negative. He also noticed increasing pigmentation of skin and oral cavity shortly.

Clinical examination revealed: pale conjunctiva, low blood pressure as $90 / 60 \mathrm{mmHg}$ with postural drop, low volume rapid pulse as $92 / \mathrm{bpm}$, temperature as $99.5^{\circ} \mathrm{F}$, some dehydration, BMI as $17.56 \mathrm{~kg} / \mathrm{m}^{2}$. hyperpigmentation of palmer creases (Figure.-1), knuckles, axilla, recent scars, buccal mucosa. With centrally placed trachea, increase in vocal fremitus, woody dull note and bronchial sound were found in left front of chest along $2^{\text {nd }}-3^{\text {rd }}$ intercostal spaces along with bilateral crackles in upper and mid areas of chest. He had left convergent squint since childhood.

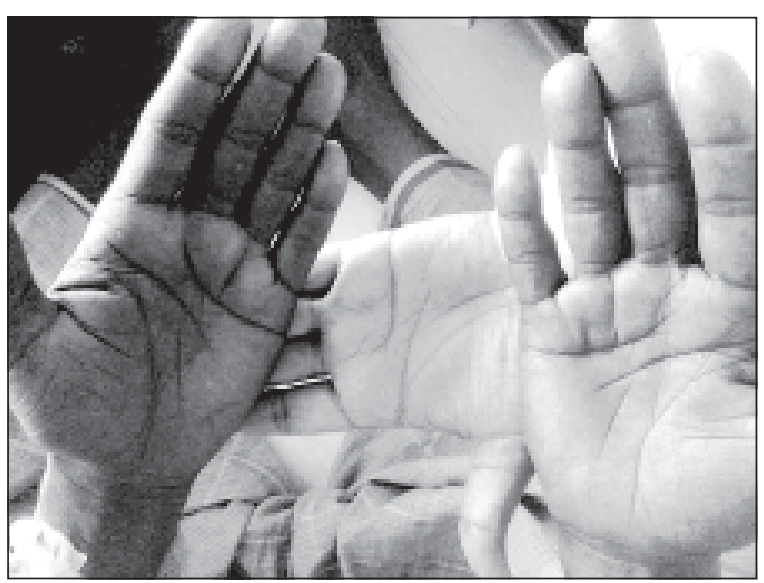

Fig.-1: Hyperpigmentation of palmer creases.

Based upon clinical scenario, investigations were done which revealed: hemoglobin $10.5 \mathrm{gm} / \mathrm{dl}$, TC 11,400/ cmm, ESR 84, CRP $12 \mathrm{mg} / \mathrm{L}, \mathrm{HbA}_{1} \mathrm{C}-6.1 \%$. hyponatraemia (126mmol/L), hyperkalaemia ( $5.7 \mathrm{mmol} /$ L), normocalcaemia $(9.3 \mathrm{mg} / \mathrm{dl})$, serum cortisol 83.44nmol/L with serum ACTH $98.0 \mathrm{pg} / \mathrm{ml}$. Liver function, renal function, serology for Hepatitis B and C, MT test, sputum for AFB (3 consecutive sample) were negative. Sputum for $\mathrm{C} / \mathrm{S}$ showed growth of $\mathrm{S}$. aureus.

To confirm the diagnosis of primary adrenocortical failure and to assess a cortisol response to stress, rapid ACTH stimulation test with Synacthen (tetracosactide $250 \mu \mathrm{gm}$ i.v.) was done which showed adrenal cortisol insufficiency (serum cortisol at ' 0 ' minute- $15.22 \mathrm{nmol} /$ L, '30' minute $52.51 \mathrm{nmol} / \mathrm{L}$ and at ' 60 ' minute -20.89 $\mathrm{nmol} / \mathrm{L})$.
Mean while chest X-ray and USG, CT of abdomen were done for further delineation of pathology. CXR showed bilateral patchy opacities in upper zones of lung fields consistent with radiological tubercular changes (Figure.2). Both USG of whole abdomen and CT of the abdomen detected bilateral enlarged adrenal glands, left one being larger $(28 \times 19 \mathrm{~mm})$, with no calcification (Figure.-3). Histopathology of CT guided FNA the Left suprarenal gland revealed blood and shadowy outline of necrotic cells.

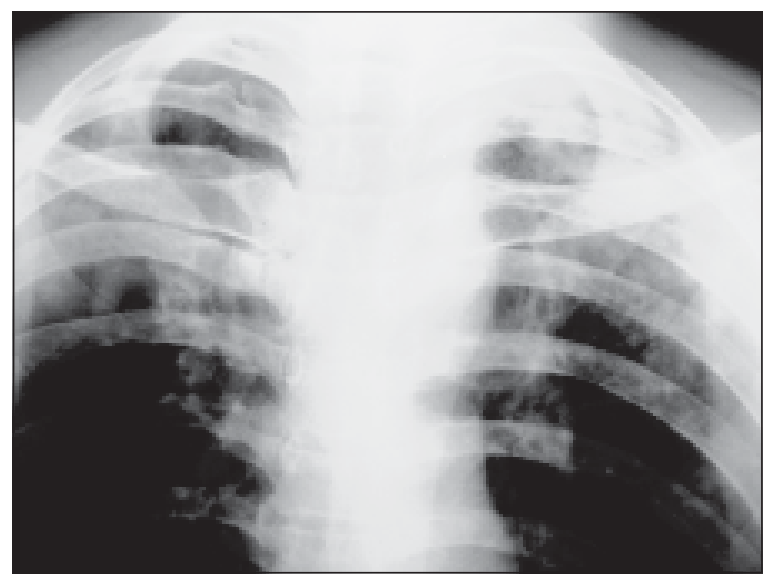

Fig.-2: Bilateral patchy opacities in both upper zones of lungs.

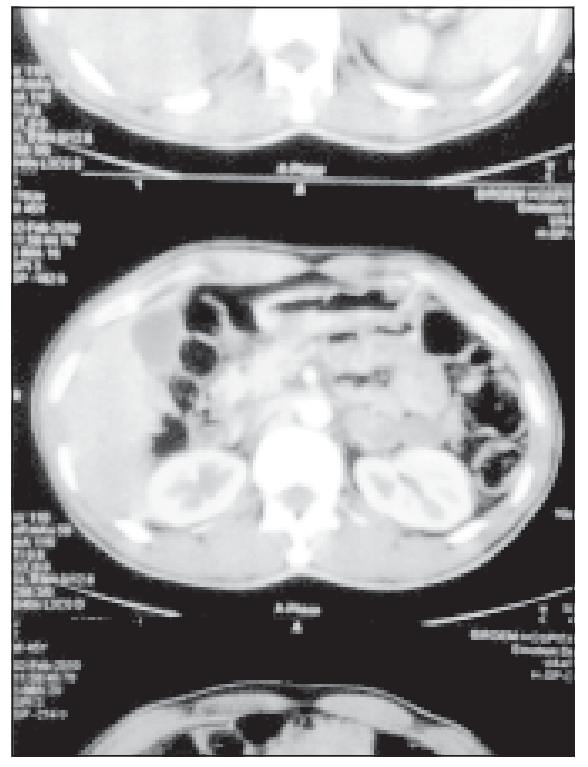

Fig.-3: $\quad C T$ abdomen shows bilateral enlagement of adrenal glands. 
Based on these difficult scenario, a diagnosis of Addison's disease due to disseminated tuberculosis was done along with super added bacterial pneumonia, DM and Left convergent squint.

\section{Clinical course of the patient:}

Initially patient was treated with $0.9 \%$ normal saline infusion, IV hydrocortisone (200mg/day), sensitive oral antibiotic (Tab.Levofloxacin and combination of Tab. Amoxicillin\& clavulonic acid). Patient's general symptoms greatly improved subsequently with standard anti-tubercular regimen. Shortly he was discharged with proper counseling and subsequent follow up revealed significant improvement of well-being, body-weight with no further event of hypoglycemia, hypotension, fever and cough.

\section{Discussion:}

Addison's disease is uncommon disease with estimated prevalence of 40-60 cases per million population. The disease can affect persons of any age, gender, or ethnicity, but typically adults between 30-50 years of age with no predispositions on ethnicity. ${ }^{9}$ Clinical presentations of Addison's disease develop insidiously. Along with typical clinical features like fatigue, postural hypotension, hypoglycemia, muscle weakness, fever, weight loss, nausea, vomiting, diarrhea etc, some have marked cravings for salt or salty foods due to the urinary losses of sodium. ${ }^{10}$ Increased pigmentation particularly at sun-exposed areas, palmar creases, sites of friction, recent scars, the vermilion border of the lips, and genital skin occurs in primary adrenal insufficiency due to increase in á-MSH which is not found in secondary and tertiary hypoadrenalism. ${ }^{11,12}$

Along with the clinical features of recurrent hypotension, hypoglycemia, progressive weight loss, increased pigmentation, our patient had hyponatremia and hyperkalaemia which were characteristic for Addison's disease. Short Synacthen test and serum ACTH supported the diagnosis. An "Addisonian crisis” or “adrenal crisis” may be precipitated by trauma, adrenal hemorrhage, intercurrent illness like infection due to insufficiency of adrenal cortical hormones to stress. ${ }^{13}$ Our case had persistent low grade fever of several months duration with evening rise of temperature, weight loss and occasional productive cough that were suggestive of pulmonary TB. Investigations suggested pulmonary TB along with add-on bacterial pneumonia that led our patient to adrenal crisis.

The CT features of active tuberculous adrenalitis associated with Addison's disease are bilateral mass like enlargement, necrosis, with or without dot-like calcification. When the contours of the adrenals are preserved, the diagnosis of infectious disease can be made with increased confidence. ${ }^{14}$ The appearance of the gland at CT depends on the chronicity of the infection and whether it has been treated or not. Among findings, most common is bilateral gland enlargement (up to 91\%), however mass like lesions are found in 50-65\% and adreniform in 35-50\%. 15, 16, 17,18 Un-enhanced CT may show attenuation commonly homogeneous, but in one third cases may be heterogeneous. ${ }^{17}$ Adrenal calcification is found in $40-60 \%$ of untreated TB patients. ${ }^{15,17}$ Nevertheless, atrophy and calcification both usually develop after treatment. ${ }^{16}$ Guo et al in their recent work on CT scan features of 42 cases of the adrenal Tuberculosis found that 91\% had bilaterally enlarged adrenals and 51\% had enlarged glands with preservation of the contours. Calcification was found in $50 \%$ cases and as the duration of Addison's disease increased, the presence of calcification and contour preservation increased concomitantly $(\mathrm{p}<0.001) .^{18}$ Bilateral cystic adrenal hyperplasia with concomitant ascites may also be found in some cases. ${ }^{19}$ Our case had bilateral adrenal enlargement with no calcification.

The diagnosis of adrenal TB is challenging. Percutaneous biopsy is proved to be of great value in determination of the final diagnosis of adrenal tuberculosis, especially when no diagnostic finding is found on CT, MRI images. ${ }^{16,20}$ Serter and associates described a 61 yearsold-man with adrenal insufficiency and an adrenal mass. The results of tuberculin skin test, sputum for AFB and culture were all negative. Only histological examination after adrenalectomy confirmed the diagnosis of TB. ${ }^{21}$ However, diagnosis may be difficult even after biopsy. ${ }^{22}$ In adrenal tuberculosis, typical granulomatous inflammation with Langhan's giant cells are found in less than half of the cases. ${ }^{22}$ The lack of granulomatous inflammation in the adrenal lesion may be related to necrosis and local suppressive effect of steroids. ${ }^{3}$ In this case, FNA revealed blood and shadowy outline of necrotic cells suggestive of necrosis of glands.

In the literature, only a few patients with tuberculosis showed recovery of adrenal function. Though adrenal 
cortex has considerable capacity of regeneration, Addison's disease due to tuberculosis is generally regarded as irreversible. ${ }^{23}$ In this case, along with standard regimen anti tubercular therapy, oral steroid was prescribed with proper counseling and patient made a good recovery.

Though presence of tuberculosis may be difficult to diagnose, it is one of the leading causes of adrenal insufficiency worldwide. ${ }^{24}$ Manifestations can be both acute and chronic. In the context of socioeconomic background and endemic pattern, high index of clinical suspicion along with judicial use and analysis of investigation modalities are required to diagnose adrenal tuberculosis.

\section{Conclusion:}

Although tubercular Addison's disease has been decreasing markedly in recent years, the possibility should be considered when weight loss, gastrointestinal symptoms, hyponatraemia and hyperkalaemia are observed in patients with active tuberculosis as well as in those having a past history of tuberculosis. The diagnosis of adrenal TB is very challenging and negative acid fast stain shouldn't denounce the diagnosis. This case presented with features of progressive primary adrenal insufficiency due to disseminated tuberculosis and finally with adrenal crisis was caused by add-on bacterial pneumonia which summoned stress on the background of chronic adrenal insufficiency.

\section{References:}

1. Addison, T. On the Constitutional and Local Effects of Disease of the Supra-renal Capsules. Highley, London 1855.

2. Husebye E, Lovas K. Pathogenesis of primary adrenal insufficiency. Best Pract Res Clin Endocrinol Metab 2009;23(2):147-57.

3. Oelkers W. Adrenal insufficiency. N Engl J Med 1996;335:1206-1212.

4. Nomura K, Demura H, Saruta T. Addison's disease in Japan: characteristics and changes revealed in a nationwide survey. Intern Med 1994; 33:602-606.

5. Brooke AM, Monson JP. Addison’s disease. Medicine 2009; 37(8): 416-419.

6. Bhatia E, Jain SK, Gupta RK et al. Tuberculous Addison's disease: lack of normalization of adrenocortical function after anti-tuberculous chemotherapy. Clin Endocrinol (Oxf) 1998; 48(3): 355-359.

7. McBrien DJ. Steatorrhea in Addison's disease. Lancet 1963;1:25-6.
8. Williams GH, Dluhy RC. Disorders of the adrenal cortex. In: Braunwald E, ed. Harrison's Principles of Internal Medicine. 17th ed. New York: McGraw-Hill Professional; 2008: 2247-2268.

9. http://emedicine.medscape.com/article/116467-overview.

10. Ten S, New M, Maclaren N. Clinical review 130: Addison's disease. J Clin Endocrinol and Metab 2001; 86 (7): 2909-2922.

11. de Herder W, van der L, Aart J. Addisonian crisis and relative adrenal failure. Reviews in Endocrine and Metabolic Disorders 2003; 4 (2): 143-7.

12. Nieman LK, Chanco T, Maria L. Addison's disease. Clinics in Dermatology 2006; 24 (4): 276-280.

13. Stewart PM. The Adrenal Cortex. In: Larsen P, ed. Williams Textbook of Endocrinology. 10th ed. Philadelphia: Saunders; 2003:491-551.

14. Wang YX, Chen CR, He GX, Tang AR. CT findings of adrenal glands in patients with tuberculous Addison's disease. J Belge Radiol 1998;81(5):226-8

15. Yang ZG, Guo YK, Li Y, Min PQ, Yu JQ, Ma ES. Differentiation between tuberculosis and primary tumors in the adrenal gland: evaluation with contrast enhanced CT. Eur Radiol 2006; 16:2031 -2036.

16. Liatsikos EN, Kalogeropoulou CP, Papathanassiou Z, et al. Primary adrenal tuberculosis: role of computed tomography and CT-guided biopsy in diagnosis. Urol Int 2006; 76: 285-287

17. Ma ES, Yang ZG, Li Y, Guo YK, Deng YP, Zhang XC. Tuberculous Addison's disease: morphological and quantitative evaluation with multi-detector CT. Eur J Radiol 2007; 62:352-358.

18. Guo YK, Yang ZG, Li Y, Ma ES, Deng YP, Min PQ, Yin LL, Hu J, Zhang XC, Chen TW. Addison's disease due to adrenal tuberculosis: Contrast-enhanced CT features and clinical duration correlation. Eur J Radiol 2006;62:126-131.

19. Patnaik MM, Deshpande, AK. Cystic adrenal hyperplasiadiagnosis? Clin Med Res 2007;5:227.

20. Yee AC, Gopinath N, Ho CS, Tao LC. Fine-needle aspiration biopsy of adrenal tuberculosis.Can Assoc Radiol J 1986;37(4):287-9.

21. Serter R, Koç G, Demirbas B, Culha C, Ongören AU, Ustün $\mathrm{H}$, Aral Y. Acute adrenal crisis together with unilateral adrenal mass caused by isolated tuberculosis of adrenal gland. Endocr Pract 2003;9(2):157-61.

22. Lam KY, Lo CY A. Critical examination of adrenal tuberculosis and a 28-year autopsy experience of active tuberculosis. Clin Endocrinol (Oxf) 2001; 54 :633-639.

23. Penrice J, Nussey SS Recovery of adrenocortical function following treatment of tuberculous Addison's disease. Postgrad Med J 1992;68(797):204-5.

24. Haddara WM, Van Uum SH. TB and adrenal insufficiency. CMAJ 2004; 171:710. 